\title{
A Benchmarking analysis of analytical and advanced nonlinear tracking control techniques
}

\author{
Samia Charfeddine* and Houssem Jerbi** \\ *National Engineering School of Gabès, Research Unit of Photovoltaic, Wind and Geothermal Systems, University of Gabès, Tunisia \\ **College of Engineering, Hail University, Department of Industrial Engineering, Kingdom of Saudi Arabia \\ *Corresponding Author: samia.charfedine@yahoo.fr
}

Submitted: 25/12/2019

Revised: $\quad 17 / 10 / 2020$

Accepted: 22/10/2020

\begin{abstract}
This paper aims at studying two advanced techniques that are used in control theory of nonlinear affine systems. These later techniques tackle the problem of the trajectory tracking with high steady state performances. The objective of this work is to compare and analyze the dynamical behavior of the desired output when controlled by the gain scheduling method and afterward with a neural control approach. Both techniques are evaluated through a numerical simulation study of a photovoltaic system, which is characterized by hard nonlinear features and challenges. The efficiency and limitations of both techniques are accurately discussed.
\end{abstract}

Keywords: Feedback linearization; Gain scheduling; Neural networks; Nonlinear control; Photovoltaic system.

\section{INTRODUCTION}

Control of nonlinear systems can be carried out by many techniques, which have been adapted in several industrial applications. Most of these techniques have a common objective of a desired output trajectory tracking (Sato \& Peaucelle, 2013; Chaouech et al., 2019, Sardellitti et al., 2013).

With the help of the digital computational methods, it is possible, to a certain extent, to overcome the limitations shown by the different analytical approaches. Amongst all the approaches, the nonlinear gain scheduling approach can be cited (Souza \& Osowsky, 2013; Dahleh et al., 2000), as it has generated a lot of interest, because of its practical efficiency. The key idea of the gain scheduling approach is to represent the original nonlinear description using a set of simple linear models (Feng, 2006; Hamdy, 2013). Hence, this technique is quite popular, when the controlled process undergoes many variations during the operation, which is an extremely regular occurrence. Likewise, it is impossible to design any global controller, which would be able to work satisfactorily in the whole state space (Palm et al., 1996). Furthermore, the controlled system performance decreased when the controller is unable to track the various changes, which marred the operational conditions. Moreover, the gain scheduling approach helps in exploiting the linear control tools for the case hardly nonlinear plants description (Charfeddine \& Jerbi, 2012). Additionally, it displays a better flexibility and a simple design for the closed loop-controlled system (Palm et al., 1996; Palm et al., 2000).

It is seen that the gain scheduling control can be synthesized by interpolating the local controllers for the stationary and the isolated states (Souza \& Osowsky, 2013; Hespanha, et al., 2007). However, some of the regulators could be ineffective due to their instability with regards to the hidden coupling and inherent uncertainties in the studied models (Souza \& Osowsky, 2013). Essentially, the heuristic implementation of this approach could ensure neither the stability nor the convergence of the controlled output. 
In this study, the gain scheduling technique is combined with the input-output linearization formalism (Isodiri, 1998; Liu \& Tong, 2015; Chemachema \& Belarbi, 2010) to enhance the performance of the gain scheduling method (Charfeddine \& Jerbi, 2012, Jebri et al., 2011).

The stability of the closed loop-controlled system is established using a non-Lyapunov stability method (Genesio et al. 1985). Additionally, the design method applies the reverse trajectory method to maximize the stability areas surrounding the operating points (Genesio et al., 1985, Jerbi, 2017).

In this study, the investigated gain scheduling strategy is based on the formalism of the state feedback exact linearization methodology and the non-Lyapunov scheme to extend operational asymptotic stability regions (Genesio et al., 1985). The major goal of this scheme consists of overcoming the limitations perceived with the input-output linearization technique as the nonminimum phase propriety characterizing many practical nonlinear plants (Yu \& Verhaegen, 2018). Hence, the performance of the approach is based on its dependence on all the equivalent models used for the different operating points (Palm et al., 1996). Decreasing the operating point number, result in an enhanced and guaranteed tracking accuracy (Charfeddine \& Jerbi, 2012).

A main motivation of this study is to carry out a comparative analysis of the enhanced gain scheduling method with a neural network control approach.

Recently, an important effort is made in designing the feedback control systems, which are seen to imitate the biological system reasoning. The "universal model-free control" have garnered a lot of interest lately, as they do not require a mathematical modeling to monitor the plant but imitate the biological process behavior for learning about the systems that they supervise on-line, hence, improving the performance automatically. The different plants include the fuzzy logic control that imitates the reasoning and linguistic functions, and the artificial neural networks, that are dependent on the biological neuronal structures having interconnected nodes. Till date, the theories and the applications of the nonlinear network structure in the feedback control are reported in the literature. It is understood that the neural networks present an extension of the adaptive control processes for the nonlinearly parameterized learning systems. The neural network tracking controller with the help of the backstepping process has been investigated in (Sadati et al., 2007; Kanellakopoulos et al., 1991).

This paper provides an evaluation framework for comparing two approaches. Hence, the continuity of the gain scheduling control performance is maintained in spite of various changes taking place in the process with respect to the modelling and primary aspects, which characterize the continuous system models having a neural control. The result of the comparative analysis is intended to pave the way for designing a novel neural network gain scheduling nonlinear control. The performance of the new strategy can be defined in terms of response swiftness and accuracy of the steady state regime while ensuring the stability of the closed loop system.

The complete paper has been organized as follows: Section 2 presents the important results of the neural network control, while Section 3 describes the analytical gain scheduling control technique. In Section 4, we described the mathematical model for the photovoltaic system. Section 5 presented the simulation study for the evaluation of the performance of the trajectory tracking. Lastly, in Section 6, we have presented all the concluding remarks.

\section{NONLINEAR GAIN SHEDULING CONTROL TECHNIQUE}

Here, the scheduling gain design is based on the input-output linearizing control method. Furthermore, the stability analysis is established according to a non-Lyapunov method for the different operating points. The arising synthetic technique used the reverse trajectory method advantages for enlarging the attraction domains surrounding the operating points (Charfeddine et al., 2013).

\section{Studied system modeling}

The class of single-input, single output systems is considered. This class is characterized by its propriety to be accurately linearizable. This class could be identified based on its representation as the state space canonical form for the following controllability: 
$\left\{\begin{array}{l}\dot{X}=F(X)+G(X) U \\ y=h(x)\end{array}\right.$

wherein $F, G: \mathfrak{R} \rightarrow \mathfrak{R}^{n}$ represent the 2 vector functions that were characterized by $F(0)=0$ and $\forall X \in \mathfrak{R}^{n},|G(X)| \geq G_{m}$.

Let us consider the variation model, which has the following description:

$\left\{\begin{array}{l}x=X-X_{n} \\ u=U-U_{n}\end{array}\right.$

wherein $X_{n}$ refers to the nominal state vector in the operating point, while $U_{n}$ is the control input variable.

Eq. (1) represents the nonlinear state model and it was easily transformed into the variation polynomial model that is as follows:

$$
\dot{x}=\sum_{i=1} f_{i} x^{[i]}+\sum_{j=0}\left(g_{j} \otimes x^{[i]}\right) u
$$

\section{Fundamentals of the gain scheduling technique}

Let us consider the system described by Eq. (1). The linearization issue using the feedback technique involves computing the smooth functions $q($.$) and p\left(\right.$.) with $q\left(X_{0}\right) \neq 0$ along with the diffeomorphism $T$ where $T\left(X_{0}\right)=0$.

For the SISO nonlinear system described by Eq. (1) and having a relative degree, $r$, in $X_{0}$ (Isodiri, 1998; Deutscher, 2005), the system can be written as

$$
\left\{\begin{array}{l}
T_{1}(X)=h(X) \\
T_{2}(X)=L_{f} h(X) \\
\vdots \\
T_{r}(X)=L_{f}^{r-1} h(X)
\end{array}\right.
$$

If the relative degree $r$ is such that, $r<n$ in $X_{0}$, the system is converted to the normal form, after determining $(n-r)$ functions, $T_{r+1}(X) \ldots T_{n}(X)$ wherein the function values for $X_{0}$ are as follows:

$$
\left\langle\frac{\partial T_{i}(X)}{\partial X}, g(X)\right\rangle=L_{g} T_{i}(X)=0
$$

For $r+1 \leq i \leq n$ in the above equation, $L_{g} T_{i}(X)$ represents the function Lie derivative for $T_{i}(X)$ with regards to $g(X)$.

Then, the diffeomorphism $T(X)$ is expressed as

$$
T(X)=\left[\begin{array}{l}
h(X) \\
L_{f} h(X) \\
\vdots \\
L_{f}^{r} h(X) \\
\xi_{1}(X) \\
\xi_{n-r}(X)
\end{array}\right]
$$


The dynamic compensator, $\xi_{i}(X)$ values with $n-r$ components can be determined using Eq. (5).

The resulting linear equivalent system characterized by the transformed variables, $Z=T(X)$ is described by Brunovsky's equivalent form given by

$$
\dot{Z}=M Z+N v
$$

wherein the pair of matrices $(M, N)$ is controllable and the linearizing manipulated variable $v$ is written as

$$
v=p(X)+q(X) U
$$

with;

$$
U=-\frac{p(X)}{q(X)}+\frac{v}{q(X)}=\alpha(X)+\beta(X) v
$$

\section{Main steps of the control algorithm}

Thereby, the scheduled gains strategy can be synthesized following a four steps algorithm:

\section{> Step 1: Synthesis and calculation of the analytical transformation}

Step 1 is mainly performed to compute the diffeomorphism:

$$
Z=T(X)
$$

The feedback is used for regulating the variation model near the origin (the regulation of the process in the operating points neighborhood is carried out in the same methodology manner).

Step 2: Synthesis of the variation model:

This step is based on the described form given by Eq. (2) and Eq. (3).

\section{Step 3: Calculation of the Dynamic feedback:}

Expressing the polynomial representation for the dynamic feedback $u$, the components of the input-output linearizing control can be computed as follows:

$$
u=-\frac{L_{f}^{r} h(x)}{L_{g} L_{f}^{r-1} h(x)}+\frac{1}{L_{g} L_{f}^{r-1} h(x)} v
$$

wherein the quantities of $L_{f}^{r} h(x)$ and $L_{g} L_{f}^{r-1} h(x)$ are expressed under the polynomial form as follows:

$$
\left\{\begin{array}{l}
L_{f}^{r} h(x)=\sum_{i=1} L_{i} x^{[i]} \\
L_{g} L_{f}^{r-1} h(x)=\sum_{j=0} J_{j} x^{[j]}
\end{array}\right.
$$

The control $v$ for the Brunovky model is determined using a simple pole placement. Hence:

$$
v=-K T(x)=-K \sum_{k=1} T_{k} x^{[k]}
$$

wherein $K=\left[\begin{array}{llll}k_{1} & k_{2} & \ldots & k_{n}\end{array}\right]$ is the control gain vector, which was determined using pole placement. Therefore, the synthesis of the polynomial dynamic feedback $u$ can be obtained by combining Eq. (11), Eq. (12) and Eq. (13) and results in 


$$
u=-\left[\frac{\sum_{i=1} L_{i} x^{[i]}}{\sum_{j=0} J_{j} x^{[j]}}+\frac{K \sum_{k=1} T_{k} x^{[k]}}{\sum_{j=0} J_{j} x^{[j]}}\right]
$$

After carrying out algebraic manipulations, the control can be written as follows:

$$
u=\sum_{i=1} \alpha_{i} x^{[i]}+\sum_{j=1} \mu_{j} x^{[j]}
$$

wherein

$$
\left\{\begin{array}{l}
\alpha_{1}=\left(J_{0}^{-1}\right) L_{1} \\
\vdots \\
\alpha_{i}=J_{0}^{-1}\left[L_{i}-\sum_{p=1}^{i-1}\left(J_{i-p} \otimes \alpha_{p}\right)\right] \quad i \geq 1
\end{array},\left\{\begin{array}{l}
\mu_{1}=\left(J_{0}^{-1}\right) K T_{1} \\
\vdots \\
\mu_{j}=J_{0}^{-1}\left[K T_{j}-\sum_{p=1}^{j-1}\left(J_{i-p} \otimes \mu_{p}\right)\right] \quad j \geq 1
\end{array}\right.\right.
$$

Replacing the $u$ by its polynomial expression of the system (15) in Eq. (3), leads to the model autonomous form, which is expressed as

$$
\dot{x}=\sum_{i=1}^{r} P_{i} x^{[i]} \dot{x}=\sum_{i=1}^{q} P_{i} x^{[i]}
$$

wherein $\left\{\begin{array}{l}P_{1}=f_{1}+g_{0}\left(\alpha_{1}+\mu_{1}\right) \\ \vdots \\ P_{i}=f_{i}+\sum_{j=0}^{i}\left(g_{j} \otimes \alpha_{i-j}\right)+\sum_{j=0}^{i}\left(g_{j} \otimes \mu_{i-j}\right) \quad i \geq 1\end{array}\right.$

\section{> Step 4: Synthesis of the output trajectory tracking control:}

In this step, the control strategy seeks a primary stability region near the operating point $X_{n_{i}}$ for a desired trajectory $y_{d}$ that ensures the asymptotic stability. Conventional methods in linear control theory provide a region of attraction, where the radius is significantly reduced for industrial processes like electric machinery and chemical reactors. This weakness imposes fixing a big number of operating points that must be studied for any desired trajectory $y_{d}(t)$. As a result, a substantial calculation time is needed to compute the control input. This restricts the implementation of the designed technique for a process with a slow dynamic $y_{d}(t)$.

To assess this weakness, a technique for regions of attraction maximization is used. (Charfeddine \& Jerbi, 2012). As a non-Lyapunov techniques the reverse trajectory method is implemented to overcome the limitations due to the computational time.

Finally, the control strategy algorithm formulated in 4 steps, as described previously, has been summarized using the following flowchart (Figure1). 


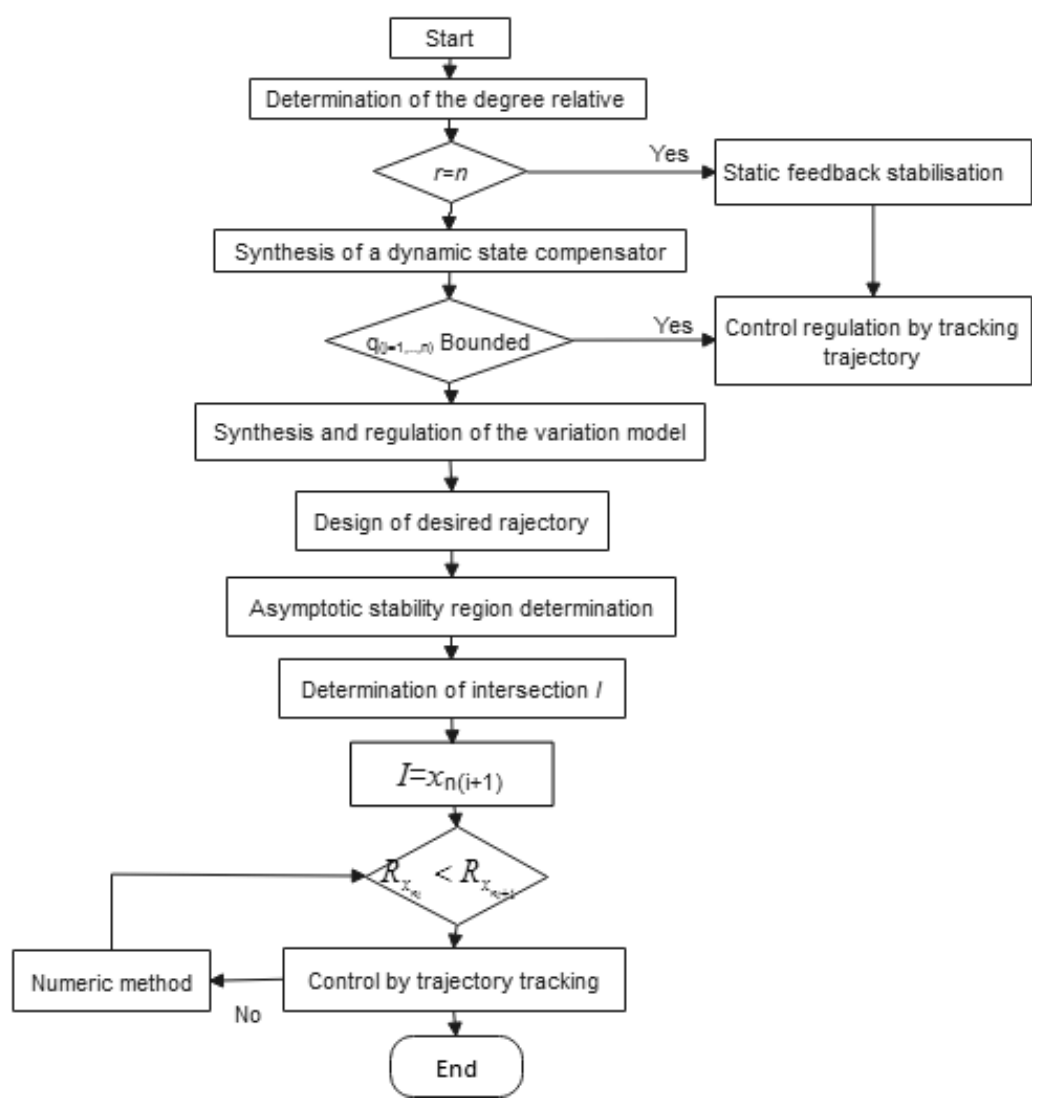

Figure 1. Flowchart of the gain scheduling control Strategy.

\section{THE NONLINEAR COMPENSATION TECHNIQUE USING NEURAL NETWORKS Selected neural network control topologies}

In the feedback control design, it is very important to ensure the tracking performance and the boundedness, or the internal stability for all the variables. If these are not ensured, serious problems can occur in the closed-loop systems, which can include the instability and the signal unboundedness, and further causes a failure of complete destruction of the system. (Werbos, 1989; Narendra \& Parthasarathy, 1990) first proposed applying the NN for the control systems. The neural network control possessed 2 main thrusts: Approximative Dynamical Programming that applies the NN for approximately solving the optimal control problems, and the neural network in a closed-loop feedback control (Narendra \& Parthasarathy, 1991). A few of these topologies have been derived from the general topologies in the adaptive control (Landau \& Silveira, 1979). Basically, there are 2 types of feedback control topologies- direct and the indirect techniques. In the case of the indirect NN control techniques, 2 functions are present; in the identifier block, the NN system is tuned for learning about the dynamics of an unknown plant, while the controller block applies this data for controlling the plant. The Direct control is seen to be more effective and it involves a direct tuning of the parameters present in the adjustable NN controller. The main limitation in applying the NN for the feedback control purpose involves the selection of a proper control system structure, followed by the demonstration of mathematicallysuitable techniques for tuning the NN weights such that the performance and the closed-loop stability are ensured (Campos \& Lewis, 1999).

In this work, the indirect topology will be exploited. An advanced tracking control method will be merged with a NN compensation. 


\section{LINEARIZATION USING THE FEEDBACK CONTROL DESIGN AND NEURAL NETWORKS}

Here, the study primarily focuses on solving the problem of nonlinear output tracking using the feedback linearization concept. A technique, which is based on process neural network modelling, will be implemented.

It is interesting to mention that all the recent studies proved that the neural control laws could guarantee the system asymptotic stability using the single-layered neural network (Sadegh, 1993).

\section{Tracking problems and error dynamics}

In this study, the linearizing feedback technique for addressing the nonlinear output tracking issues is exploited. The objective is as follows:

Let us consider the required output $y_{d}(t)$. Thereafter, the output tracking design consists of determining the control law $u$, which allows the process output to track the desired trajectory with a satisfactory steady state behavior and asymptotic stability.

Let us consider the vector $x_{d}(t)$, which is defined as

$x_{d}(t)=\left[y_{d}, \dot{y}_{d}, \ldots y_{d}^{n-1}\right]^{T}$

Furthermore, it is also assumed that the vector $x_{d}$ is measurable with the below property:

$\left\|x_{d}(t)\right\| \leq Q$

let us determine the state vector error as $e=x-x_{d}$, while the filtered error is

$$
\eta=\left[\Gamma^{T} l\right] e \quad\left[\Gamma^{T} l\right]
$$

wherein $\Gamma=\left[\lambda_{1}, \lambda_{2}, \ldots, \lambda_{n-1}\right]$ is the vector with coefficients that are randomly selected such that the error, $e$, tends exponentially towards 0 when the filtered error, $\eta$ tends towards 0 .

The filtered error derivatives are as follows:

$$
\dot{\eta}=f(x)+g(x) u+Y_{d}
$$

wherein $Y_{d}=-y_{d}^{(n)}+\left[0 \Gamma^{T}\right] e\left[0 \Gamma^{T}\right]$

\section{Neural network structure}

The neural network equation is represented as follows:

$$
h(x)=W^{T} \sigma\left(V^{T} x\right)+\varepsilon_{L_{k}}(x)
$$

where $W \in \mathfrak{R}^{L_{h}+1}$ and $V \in \mathfrak{R}^{(n+1)^{*} L_{k}}$ represented the weights of the neural network, $L_{h}$ was the no. of neurons present in the hidden layer while $\varepsilon_{L_{h}}(x)$ referred to the error term, which was dependent on the $L_{k}\left(\varepsilon_{L_{h}}(x)\right.$ and decreased with an increase in $L_{k}$.

A network threshold was introduced in the vector $x$, and also, the activation function selected for the neurons in the layer corresponded to the general sigmoid function that was used frequently in the earlier published studies. 
The weight generalized matrix is defined as

$$
\Upsilon_{h}=\left[\begin{array}{cc}
V_{h} & 0 \\
0 & W_{h}
\end{array}\right]
$$

Practically, the weight of the network $W_{h}$ and $V_{h}$, were seen to be bounded by the constant parameter, which helped in stating that

$$
\left\|\Upsilon_{h}\right\| \leq \Upsilon_{h m}
$$

wherein $\Upsilon_{h m}$ was a given parameter.

The concept of limiting the network weights was seen to be developed from the robust control processes. But, despite the absence of any algorithm, which specified the terminal $\Upsilon_{h m}$, this was a reasonable assumption made in the available neural network literature.

\section{Synthesis of the neural control law}

In this section, the function $f($.$) and g($.) will be estimated using neural network design. Therefore, the control law was determined as

$$
u=\frac{1}{\hat{g}\left(\hat{\Upsilon}_{g}, x\right)}\left[-\hat{f}\left(\hat{\Upsilon}_{f} x\right)+v\right]
$$

wherein the estimates $\hat{f}\left(\hat{\Theta}_{f}, x\right)$ and $\hat{g}\left(\hat{\Theta}_{g}, x\right)$ are the estimated function $f($.$) and g($.$) and the auxiliary term v$ was expressed as

$$
v=-K_{v} \eta-Y_{d}
$$

It can be seen that $\hat{g}\left(\hat{\Theta}_{g}, x\right)$ cannot be 0 , hence, a technique for bounding the control signal while implementing the linearizing feedback input will be used. In Figure 2, the overall control scheme is shown.

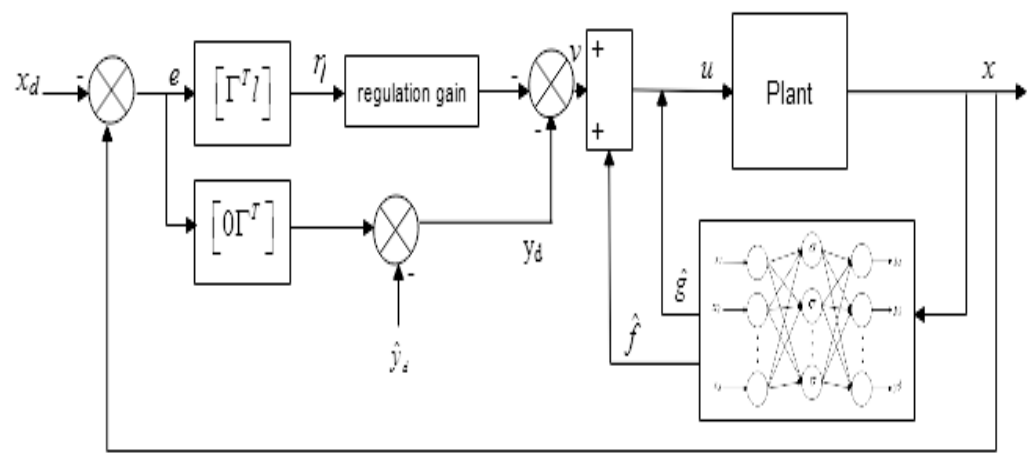

Figure 2. The closed loop control structure using the neural networks.

\section{SIMULATION ANALYSIS}

This section is reserved to study the control problem of a photovoltaic system (Andoulssi et al. 2013) using the gain scheduling technique and the neural network approach, which are both presented in the previous sections. This section will start with the photovoltaic model description than the implementation of both control techniques will be addressed. 


\section{Description of the system}

Figure 3 describes the functional diagram for the considered photovoltaic system. This system comprises a DC / DC reducing converter, a photovoltaic generator, and a DC motor pump.

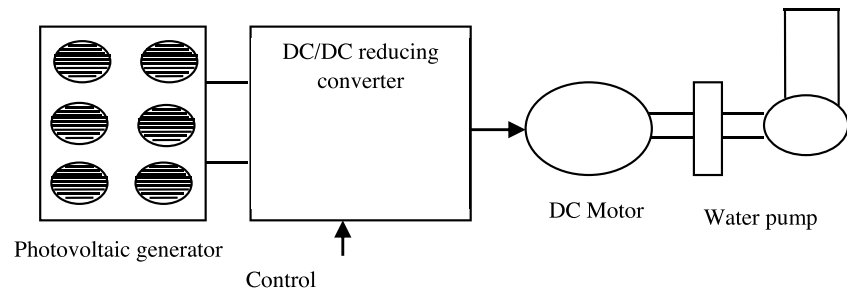

Figure 3. Functional diagram of the considered photovoltaic system.

\section{Photovoltaic generator model}

In general, a diode model is used to represent the electrical behavior of the photovoltaic cell. In the present model, the photovoltaic cell that uses the electric current generator is represented. This description is equivalent to a current source that is parallel to the diode.

Similar to the junction diode, the photovoltaic diode also has a nonlinear current-voltage relationship (Miamouni et al., 2004 Andoulsi et al., 1999). The current is linked to the solar generator voltage by the following equation:

$$
I_{p}=I_{p h}-I_{S}\left[\exp \left(\frac{V_{p}}{V_{T}}\right)-1\right]
$$

wherein $I_{p}$ and $V_{p}$ represent, respectively, the current and output voltage.

The model remaining parameters are listed in Table 1. The current PV generator includes 2 parallel connecting channels $(50 \mathrm{~W})$. Each channel contains 4 panels in series, where every panel consists of 36 connected cells (Figure 4).

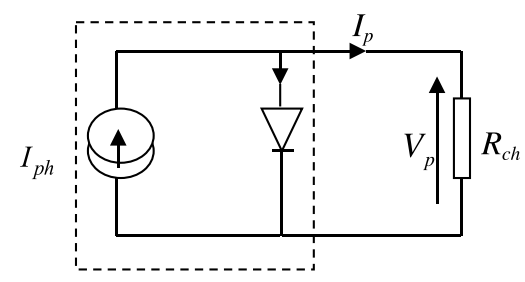

Figure 4. Equivalent circuit of an ideal solar cell.

Table 1. Different parameters.

\begin{tabular}{|l|l|}
\hline$V_{T}=\frac{n K_{B} T}{q}$ & Thermodynamic potential \\
\hline$I_{p h}$ & Pv generator photocurrent proportional to the irradiance level \\
\hline$I_{S}$ & Reverse current of the diode saturation of a photovoltaic cell. \\
\hline $\mathrm{z} q$ & Electron charge \\
\hline$T$ & Temperature of the solar panel $\left({ }^{\circ} \mathrm{K}\right)$ \\
\hline$n$ & The ideal PN junction factor \\
\hline$K_{B}$ & Constant of Boltzman $\left(1,38.10^{-23} j /{ }^{\circ} \mathrm{K}\right)$ \\
\hline
\end{tabular}




\section{Converter model}

A solar generator is a form of a nonlinear process, which generates a maximal power at a particular operating voltage and current point. To control the delivered power to the Dc motor pump a DC-DC converter is inserted in series between the pump and the PV generator. Figure 5 shows the circuit diagram for the considered photovoltaic system.

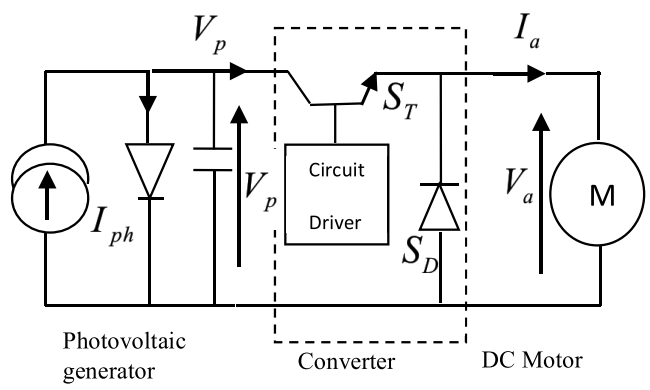

Figure 5. Equivalent circuit diagram of the photovoltaic system.

It can be noted that the convertor contains a circuit control driver related to power transistor $\left(S_{T}\right)$ along with the free-wheel diode $\left(S_{D}\right)$. The generated power is transmitted to the load using the switching transistor $S_{T}$ that is turned on / off periodically using an external control circuit, identified as the Pulse Width Modulation (PWM). Eq. (26) is describes the mean output voltage:

$$
V_{a}=\rho V_{p}
$$

wherein $V_{p}$ and $V_{a}$ represent the output and the converter-input voltage, respectively, and $\rho$ refers to a switch cycle. Based on Eq. (26), it is obvious that the output voltage can be controlled by varying the $\rho$ value of the chopper duty cycle. For varying $\rho$, the PWM design control of the process is applied (Packiam et al. 2015). Assuming the low power loss assumption, where the photovoltaic generated power is equivalent to the load consumed power, it comes:

$$
\begin{aligned}
& V_{p} I_{p}=V_{a} I_{a} \\
& \frac{V_{p}}{V_{a}}=\frac{I_{a}}{I_{p}}=\rho
\end{aligned}
$$

\section{DC motor pump model}

The DC motor pump is a designed as a simple permanent magnet machine. In this study, it is assumed that the constant flow is present for all the operational points. In the machine model, $R_{a}$ and $R_{L}$ represent the resistance and the inductance. Furthermore, the equation below describes the transfer of energy between the electrical and the mechanical parts, which is shown as a relative proportion between the f.e.m. and the angular velocity:

$$
E_{a}=k_{b} \Omega
$$

The DC motor is attached to a centrifugal pump that for simplicity is described by the couple:

$$
\Gamma=k_{T} \Omega
$$


Therefore, the photovoltaic system model is written as

$\left\{\begin{array}{l}\dot{i}_{a}=-\frac{R_{a}}{L_{a}} i_{a}-\frac{k_{b}}{L_{a}} \Omega+\frac{1}{L_{p}} v_{p} \cdot u \\ \dot{\Omega}=-\frac{k b}{J} i_{a}-\frac{k_{T}+F}{J} \Omega \\ \dot{v}_{p}=-\frac{u}{C} i_{a}-\frac{I_{s}}{C}\left[\exp \left(\frac{v_{p}}{V_{T}}\right)-1\right]+\frac{I_{p h}}{C}\end{array}\right.$

Writing system of Eq. (32) under the standard form yields

$\left\{\begin{array}{l}\dot{x}=f(x)+g(x) u \\ y=h(x)\end{array}\right.$

Wherein $x=\left[\begin{array}{l}i_{a} \\ \Omega \\ v_{p}\end{array}\right]$ is the state vector comprising the motor current, motor speed and photovoltaic generator voltage.

Hence, one has

$$
f(x)=\left[\begin{array}{l}
-\frac{R_{a}}{L_{a}} i_{a}-\frac{k_{b}}{L_{a}} \Omega \\
-\frac{k_{b}}{J} i_{a}-\frac{k_{T}+F}{J} \Omega \\
-\frac{I_{s}}{c}\left[\exp \left(\frac{v_{p}}{V_{T}}\right)-1\right]+\frac{I_{p h}}{C}
\end{array}\right], g(x)=\left[\begin{array}{l}
v_{p} \\
0 \\
-i_{a}
\end{array}\right] \text { and } y=h(x)=v_{p}
$$

Estimating the relative degree $r$ by deriving the equation output $y$ until the control $u$ appears:

$$
\begin{aligned}
\dot{y}=v & =\left[-u \cdot i_{a}-I_{s}\left[\exp \left(\frac{v_{p}}{v_{T}}\right)-1\right]+I_{p h}\right] \\
& =L_{f} h(x)+u \cdot L_{g} h(x)
\end{aligned}
$$

The control input appears from the first derivation, which concludes the relative degree of the photovoltaic system is $r=1$.

Consequently, the control input can be written in the following form: $u=\alpha(x)+\beta(x) v$

Wherein $L_{g} h(x)=\frac{\partial h}{\partial x} g(x)=-\frac{i_{a}}{C}$

When the form of the dynamic feedback $u$ polynomial equation is obtained, it is possible to establish the expression of the input-output linearizing control that yields:

$$
\begin{aligned}
u & =\frac{1}{L_{g} h(x)}\left(-L_{f} h(x)+v\right) \\
& =\frac{1}{i_{a}}\left[\left[-I_{s} \exp \left(\frac{v_{p}}{v_{T}}\right)-1\right]+I_{p h}\right]-\frac{C}{i_{a}} v
\end{aligned}
$$


The main control achievement involves maintaining the optimal voltage for photovoltaic generator $\left(y(t)=v_{p}\right)$.

Neglecting the reference variation, the objective can be fulfilled by selecting the control under the following form:

$v=k\left(y_{d}-y\right)$

wherein $k$ refers to the control gain vector, which can be determined with a pole placement design.

Truncating the polynomial development to the third order for the photovoltaic model, and considering the nominal operating points $\left(X_{n}, U_{n}\right)$, leads to the polynomial model below:

$\dot{x}=f_{1} x+f_{2} x^{[2]}+f_{3} x^{[3]}$

Furthermore, the polynomial form of the diffeomorphism truncated to the third order can be written as:

$$
T(x)=T_{1} x+T_{2} x^{[2]}+T_{3} x^{[3]}
$$

Finally, the manipulated variable $u$ is given by

$$
u=\alpha_{1} x+\alpha_{2} x^{[2]}+\alpha_{3} x^{[3]}+\mu_{1} x+\mu_{2} x^{[2]}+\mu_{3} x^{[3]}
$$

\section{Simulation results}

Table 2 presents the numerical values for the different parameters studied. The objective of this section is to evaluate the performance and efficiency of the studied control methods. The addressed control problem focuses on the tracking problem of a photovoltaic power system. In this study, all simulations are carried out using the MATLAB 2018 software.

Table 2. Numerical values of the model parameters.

\begin{tabular}{|l|l|}
\hline \multirow{5}{*}{ Photovoltaic Generator } & $I_{p h}=4,4 \mathrm{~A}$ \\
\cline { 2 - 3 } & $I_{S}=52,75.10^{-6} \mathrm{~A}$ \\
\cline { 2 - 3 } & $\mathrm{V}_{T}=6.73 \mathrm{~V}$ \\
\cline { 2 - 2 } & $C=4000.10^{-6} \mathrm{~F}$ \\
\hline \multirow{5}{*}{ DC Motor } & $R_{a}=1.072$ \\
\hline & $L_{a}=0.05$ \\
\hline & $J=476.10^{-6}$ \\
\hline & $F=88.10^{-5}$ \\
\hline & $K_{T}=14.10^{-4}$ \\
\hline & $K_{b}=45.10^{-3}$ \\
\hline
\end{tabular}

The first step of this study focused on the evaluation of the local performance of both controllers. The variation model represented by equation (37) is controlled to evaluate the local performance around an operating point. The state variables $x_{1}, x_{2}$ and $x_{3}$, are depicted in Figures 6, 7 and 8 . 


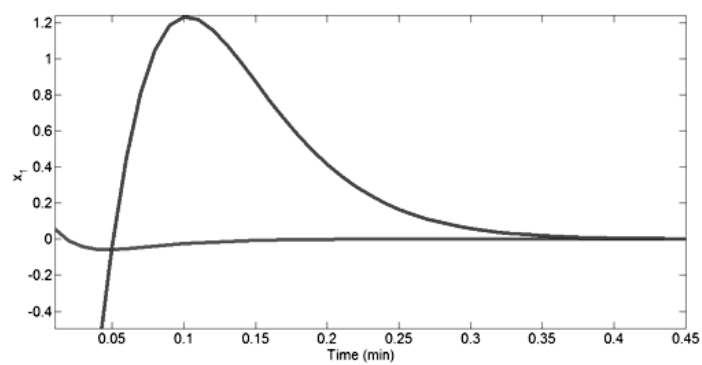

Figure 6. Dynamics of the variables $x_{1}$ (red): Neural case, (blue) Scheduling case).

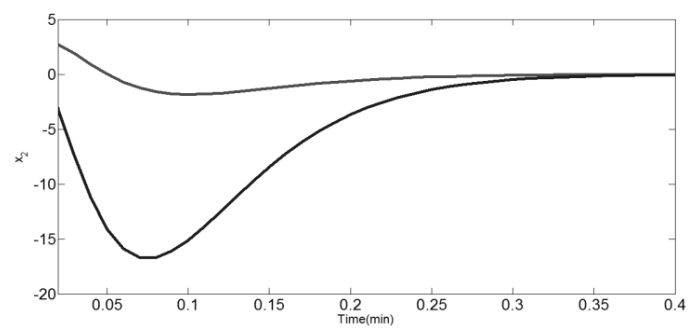

Figure 7. Dynamics of the variable $x_{2}$ ((red): Neural case, (blue) Scheduling case).

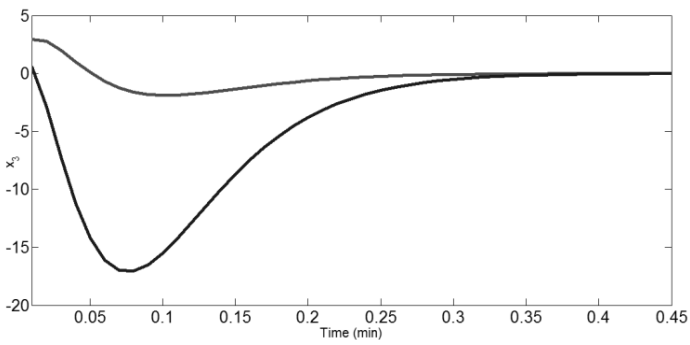

Figure 8. Dynamics of the variable $x_{3}$ ((red): Neural case, (blue) Scheduling case).

In these figures, the convergence of the state variables is ensured by both control techniques. The overall performance is quite satisfactory; with the state variables' dynamics being asymptotically stable with a swift transitory response and accurate steady state regime.

The figures have highlighted the acceptable dynamic behavior with a realistic the manipulated variable regulation. Likewise, it can be seen that the controlled variables for both cases are attracted towards the equilibrium and their dynamics does not indicate an inadmissible excess and overshooting. However, in terms of swiftness, a higher speed is noted for the scheduling gain-controlled variables. This result can be explained by the assumptions made while selecting the model truncating order and sampling time selection. Additionally, the feedback linearizing technique is very effective in compensating the plant nonlinearities as compared to the polynomial neural network.

Figure 9 represents the control inputs. The latter manage to stabilize the system dynamics within a limited time period with a reduced control effort. In particular, the neural network stabilizes the system dynamics within 0.2 minutes; however, the scheduling control stabilizes the state variables within 0.3 minutes. Table 3 summarizes the key parameters that were obtained by qualitatively analyzing the response times for the transient and steady-state responses. regime. The scheduling approach offers a settling time of $3 \mathrm{~s}$, while the neuronal approach displayed a settling time of $0.12 \mathrm{~s}$.

Likewise, the gain scheduling technique helped in achieving a rise time in $0.2 \mathrm{~s}$ however the rise time is $0.09 \mathrm{~s}$ for the neural control approach. 


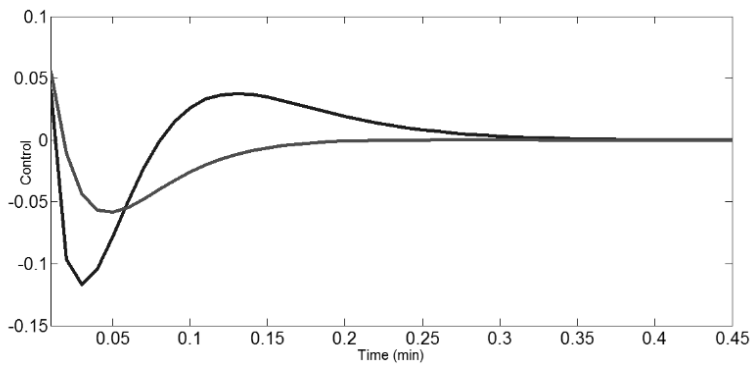

Figure 9. Control input dynamics $u$ ((red): Scheduling case, (blue) Neural case).

Table 3. Numerical results of the qualitative comparative study.

\begin{tabular}{lll}
\hline & Gain Scheduling approach & Neuronal approach \\
\hline Settling Time & $0.3 \mathrm{~s}$ & $0.12 \mathrm{~s}$ \\
Overshooting & No & No \\
Rise Time & $0.2 \mathrm{~s}$ & $0.09 \mathrm{~s}$ \\
\hline
\end{tabular}

As a first outcome the neural control approach displayed better local elementary results as compared to the gain scheduling approach. Furthermore, satisfactory dynamical results were noted with a smoother response time for the model state variables.

In the next stage of this analysis study, the learning process based on the multilayer neural networks and using the gradient approach to assess the weights by decreasing the costs will be addressed.

Here, the learning process is known as the learning with the error retro-progression. For carrying out this process and conducting the online learning, the following steps are used:

- The structure of the neural networks is shown in Figure 1

The estimation of the error is performed using an approach that decreases the output squared learning error. As a matter of fact, the error can be defined as: $E=\{\|x-\hat{x}\|\}$

Figure 10 presents the simulation results. It can be seen that the quadratic error for all the state variables tends towards 0 ; hence, the system stability can be guaranteed by applying the neuronal method.

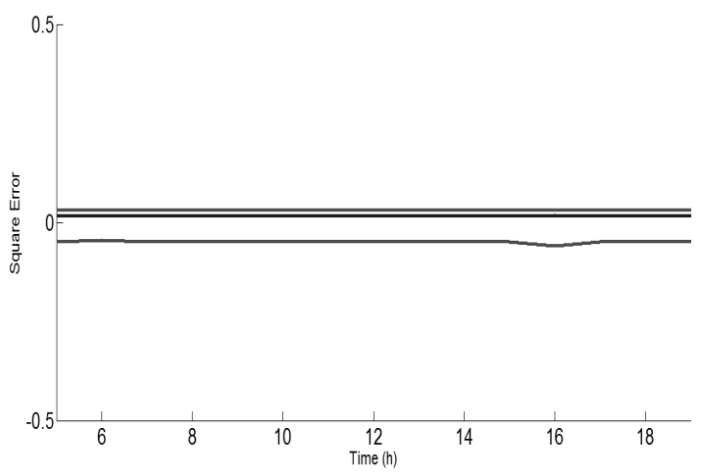

Figure 10. Square error for the different states. 
The final stage of this study is reserved for the key studied problem in this paper, which is the trajectory tracking control problem. The desired output trajectory is shown in Figure 11. This trajectory defines a standard physical behavior in terms of the system characteristics and features.

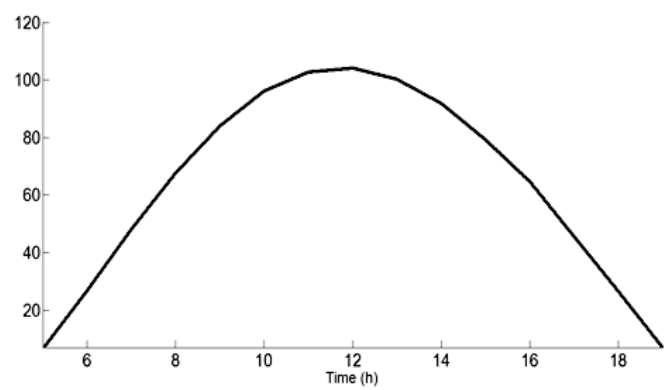

Figure 11. Desired output trajectory.

After carrying out the simulation study, the results are presented in Figure 12. A perfect agreement can be noted for all different trajectories.

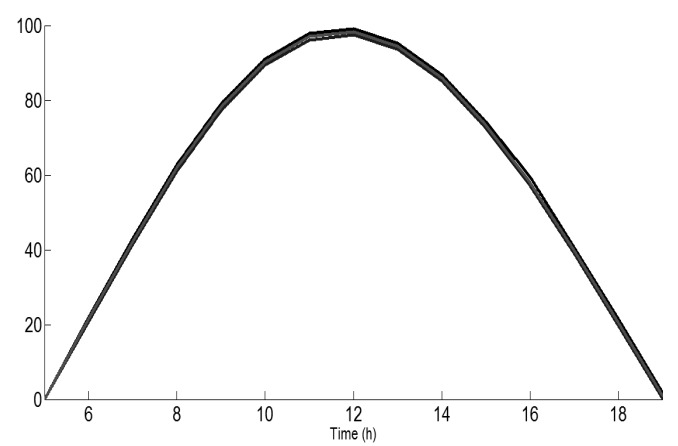

Figure 12. Output tracking trajectory ((black): Desired output trajectory, (red): Neural output trajectory, (blue): Gain scheduling output trajectory).

Correspondingly, the transient responses show that the real outputs for both techniques converge towards the desired output trajectory while the behaviour of the controlled variables does not show an inadmissible dynamic.

To emphasize the performances of the gain scheduling and the neuronal approaches, it is interesting to note that the tracking error is less than $2 \%$ for the scheduled gain approach. However, for the neural control, an error of $<1.6 \%$ was measured. This result is depicted in Figure 13.

The controllers were evaluated in real time. Therefore, the total time for carrying out these tasks did not greatly affect the system variable dynamics or the desired output trajectory.

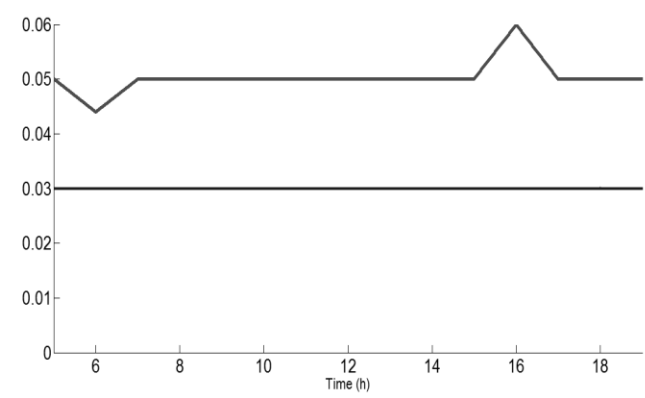

Figure 13. The tracking error ((blue): neuronal approach, (red): gain scheduling approach). 
Figure 14, describes the control input dynamics for the studied approaches. The control signals dynamics are physically satisfactory. The figure showed that there was no undesired overshoot or an undesirable saturation for both approaches. Similarly, a limited control effort for the system state variable stabilization can be noted.

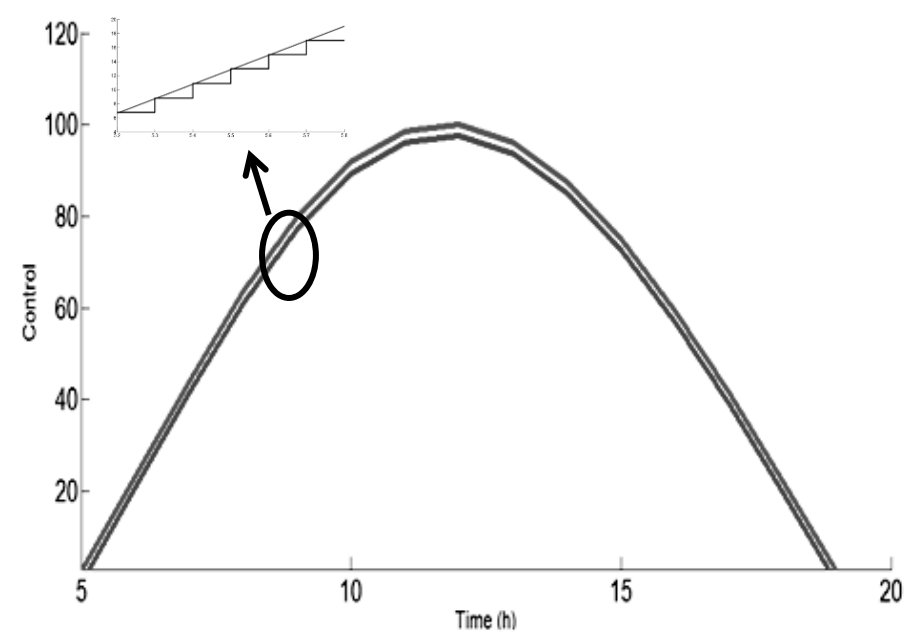

Figure 14. Control inputs dynamics ((blue): Neuronal case, (red): Scheduling case).

It is perceived that the tracking error was quite weak; however, a tracking error is still seen in this study. Moreover, this tracking error did not show a significant physical effect. Decreasing its value can be assured by increasing the number of the operating points on the desired output trajectory. These overall results helped in concluding that the studied approaches are comparable in performance level and practical efficiency.

Likewise, it can be also observed that the neural approach showed better results than the gain scheduling method. This is due to an enhanced methodology in selecting of controller gains by combining the subsequent operating points.

\section{CONCLUSIONS}

In this paper, a critical comparative analysis of an analytical approach with an advanced computing control technique is performed. The performance analysis is conducted for a PV solar power plant. Recent scheduled gain control theory is efficiently exploited as an analytical control strategy. Such theory helped assess and compute the operating state feedback gains of a stabilizing controller, which contributes to bringing the power electrical system to its nominal operating conditions following desired output trajectory constraints. The gain scheduling approach relied on the formalism of feedback linearization, gain scheduling methodology, variation based-model concept, and a nonLyapunov stability method. It can be assumed that the gain scheduling technique can fulfil the objective of a trajectory tracking with high performance standard. Furthermore, it could be seen that it was conceptually simple; therefore, it could be easily applied.

The simulation results reflect that the designed controller offers enhanced dynamic performances than various available methods in the literature. This is in terms of attenuated oscillations, minimum settling time numerical values, peak undershoot, peak overshoot, various performance indices and minimum numerical values for damping ratio. The maximization of the attraction domain around equilibrium points substantiates the output dynamic stability behavior of the systems compared to several prevalent control techniques. Nevertheless, the gain scheduling approach, due to the drawback of the linearization approach, ensures the design strategy efficiency only in regions, which are relatively close to the operating points.

On the other hand, the neuronal control approach displayed satisfactory results in recovering and compensating the hard nonlinearities of the studied plant. However, it has restrictions related to the training phase and its data structure. 
Indeed, several parameters must be taken into account as the prediction of the operating points, the dynamical stability behavior and the optimal control gains.

- Lastly, the following practical points can be addressed as further extensions for developing this work: it is promising to estimate uncertain states and different model parameters for implementing the control laws and ensuring the fault detection with disturbances rejection.

\section{REFERENCES}

Andoulsi, R., Mami, A., Dauphin-Tanguy, G. \& Annabi, M. 1999. Modelling and simulation by bond graph technique of a DC motor fed from a photovoltaic source via MPPT boost converter. Proceeding of CSSC, 99: 4181-4187.

Andoulssi, R., Draou, A., Jerbi, H. 2013. Nonlinear control of a photovoltaic water pumping system. Energy Procedia. 42: 328336.

Campos, S. \& Lewis, F.L., 1999. Dead zone compensation in discrete time using adaptive fuzzy logic. IEEE Transactions on Fuzzy Systems. 7(6): 697-707.

Chaouech, H., Charfeddine, S., Ouni, K., Jerbi, H. \& Nabli, L. Intelligent supervision approach based on multilayer neural PCA and nonlinear gain scheduling. NCAA: Neural Computing and Applications. 31(3):1-11.

Charfeddine, S. \& Jerbi, H. 2012. A Survey on nonlinear gain scheduling design control for continuous and discrete time. International Journal Modelling, Identification and Control. 19(3): 203-216.

Charfeddine, S. \& Jerbi, H. 2012. Trajectory tracking and disturbance rejection for nonlinear periodic process: A gain scheduling design. IREMOS: International Review on Modelling and Simulations. 5(2): 1075-1083.

Charfeddine, S., Jerbi, H. \& Sbita, L. 2013. Non-Linear Discrete -Time Gain Scheduling Control for Affine Non-Linear Polynomial Systems. IREMOS: International Review on Modelling and Simulations. 6(3): 1031-1041.

Chemachema, M. \& Belarbi, K. 2010. State feedback linearization-based neural network adaptive control for a class of uncertain SISO nonlinear systems. International Journal Modelling, Identification and control. 11(1-2): 44-51.

Dahleh, M.A., McConley, M.W., Appleby, B.D. \& Feron, E. 2000. A computationally efficient lypunov-based scheduling procedure for control of nonlinear systems with stability guarantees. IEEE Transactions Automatic Control. 45(1): 33-49.

Deutscher, J. 2005. Input-output linearization of nonlinear systems using multivariable Legendre polynomial. Automatica. 41(2): 299-304.

Feng, G. 2006. A survey on analysis and design of model-based fuzzy control systems. IEEE Transactions on Fuzzy Systems. 14(5): 676-697.

Genesio, R., Tartaglia, M. \& Vicino, A. 1985. On the estimation of asymptotic stability regions: State of the art new proposals. IEEE Transactions on the Automatic Control. 30(8): 747-755.

Hamdy, M. 2013. State observer based dynamic fuzzy logic system for a class of SISO nonlinear systems. International Journal of Automation and Computing. 10(2): 118-124.

Hespanha, J.P., Naghsh, Tabrizi, P. \& Xu, Y. 2007. A survey of recent results in networked control systems. Proceedings of the IEEE. 95(1): 138-162.

Isodiri, A. 1998. Nonlinear Control Systems. $2^{\text {nd }}$ ed. New York: Springer.

Jebri, W., Jerbi, H. \& Abdelkrim, M.N. 2011. Nonlinear state feedback design for continuous polynomial systems. International Journal of Control, Automation and Systems. 9(2): 566-573.

Jerbi, H. 2017. Estimations of the domains of attraction for classes of nonlinear continuous polynomial systems. Arabian Journal for Science and Engineering. 42(7): 2829-2837.

Kanellakopoulos, I., Kokotovic, P.V. \& Marino, R. 1991. An extended direct scheme for robust adaptive nonlinear control. Automatica, 27(2): 247-255.

Landau, I. \& Silveira, H. 1979. A stability theorem with applications to adaptive control. IEEE Transactions on Automatic Control. 24(2): 305-312. 
Liu, Y. \& Tong, S. 2015. Adaptive fuzzy control for a class of unknown nonlinear dynamical systems. Fuzzy Sets and Systems. 263: $49-70$.

Miamouni, M.F., Mansori, M.N., Benghanem, B. \& Annabi, M. 2004. Vectoriel command of an asynchronous motor fed by photovoltaic generator. Renewable Energy. 29: 433-442.

Narendra, K.S. \& Parthasarathy, K. 1990. Identification and control of dynamical systems using neural networks, IEEE Transactions Neural Networks. 1(1): 4-27.

Narendra, K.S. \& Parthasarathy, K. 1991. Gradient methods for the optimization of dynamical systems containing neural networks, IEEE Transactions Neural Networks. 2(2): 252-262.

Palm, R., Driankov, D. \& Rehfuess, U. 1996. A Takagi-Sugeno fuzzy gain-scheduler. Proceedings of the $5^{\text {th }}$ IEEE International Conference, Fuzzy Systems. 2: 1053-1059.

Palm, R., Stutz, C. \& Runkler 2000. A control sequence generator for fuzzy gain schedulers, Proceedings of the $39^{\text {th }}$ IEEE Conference Decision and Control. 1: 370-375.

Packiam, P., Jain, N.K. \& Singh, I.P. 2015. Steady and transient characteristics of a single stage PV water pumping system. Energy Systems. 6: 173-199.

Sadegh, N. 1993. A perceptron network for functional identification and control of nonlinear systems, IEEE Transactions Neural Network, 4: 982-988.

Sadati, H., Sabzehparvar, M., Menhaj, M.B. \& Bahrami, M. 2007. Backstepping controller design using neural networks for a fighter aircraft. European Journal of Control. 13(5): 516-526.

Sardellitti, I., Medrano-Cerda, G.A., Tsagarakis, N., Jafari, A. \& Caldwell, D.G. 2013. Gain-scheduling control for a class of variable stiffness actuators based on lever mechanisms. IEEE Transactions on Robotics. 29(3): 791-798.

Sato, M. \& Peaucelle, D. 2013. Gain-scheduled output-feedback controllers using inexact scheduling parameters for continuoustime LPV systems. Automatica. 49(4): 1019-1025.

Souza, C.E. \& Osowsky, J. 2013. Gain-scheduled control of two-dimensional discrete-time linear parameter-varying systems in the Roesser model. Automatica. 49(1): 101-110.

Werbos, P.J. 1989. Maximizing long-term gas industry profits in two minutes in lotus using neural network methods. IEEE Transactions Systems Man. Cybernetics. 19(2): 315-333.

Yu, C. \& Verhaegen, M. 2018. Data-driven fault estimation of non-minimum phase LTI systems. Automatica. 92: 181-187. 